\title{
REDES GLOBAIS E DINÂMICAS REGIONAIS. EXPLORANDO O DESENVOLVIMENTO DAS TECNOLOGIAS BI-COMBUSTÍVEL EM SÃO PAULO
}

LUÍS CARVALHO ${ }^{1}$

\begin{abstract}
Resumo - Uma importante parte do trabalho recente da geografia económica tem sido dedicado a conciliar noções de "aprendizagem territorializada" com a evidência de que muitos dos esforços de criação de conhecimento e inovação de actores locais se processam cada vez mais através de redes globais. Todavia, tem sido dada menor atenção aos efeitos dinâmicos que resultam da participação de actores localizados, nomeadamente empresas, em redes globais de conhecimento e inovação. Numa perspectiva evolucionista, este artigo propõe uma conceptualização desses efeitos ao nível da empresa, das redes regionais de inovação e co-evolução institucional associada. Esta conceptualização é ilustrada com o caso do desenvolvimento do portfólio de tecnologias bi-combustível em São Paulo, Brasil.
\end{abstract}

Palavras-chave: Redes globais, dinâmicas de conhecimento regionais, geografia económica evolucionista, bio-combustíveis, Brasil.

\begin{abstract}
Global NETWORKS AND REGIONAL DYNAMICS: EXPLORING THE DEVELOPMENT OF BI-FUEL TECHNOLOGIES IN SÃo PAULO. Recent work within economic geography has been focusing on the reconciliation of the concept of localized learning with the evidence that long-distance knowledge networks are increasingly relevant for the innovation efforts of firms. However, less attention has been paid to the dynamic effects that may spur from plugging into global knowledge networks. Taking an evolutionary perspective, this article proposes a conceptualization of these issues at the levels of the firm, of regional networks and of institutional co-evolution. It illustrates the proposed theoretical framework with the case of the new bi-fuel technological developments in São Paulo, Brazil.
\end{abstract}

Key words: Global networks, regional knowledge dynamics, evolutionary economic geography, bio fuels, bi-fuel vehicles, Brazil.

Recebido: 03/11/2008. Revisto: 05/01/2009. Aceite: 12/01/2009.

1 EURICUR - European Institute for Comparative Urban Research Erasmus University Rotterdam. E-mail: decarvalho@ese.eur.nl. 
Résumé - RÉSEAUX MONDIAUX ET DYNAMIQUES RÉGIONALES: DÉVELOPPEMENT Des techniques liÉes aux doubles-Combustibles, À SÃo Paulo (BRÉSil). De nombreuses recherches récentes en Géographie économique tentent de concilier la notion d'apprentissage dans un cadre territorial déterminé, avec le fait que les efforts d'innovation sont de plus en plus basés sur des réseaux à l'échelle mondiale. Mais les effets dynamiques qui résultent de l'insertion des entreprises d'une région donnée dans les réseaux mondiaux de connaissance et d'innovation, ont encore été insuffisamment étudiés. On tente ici de conceptualiser ces effets, tant au niveau des entreprises qu'à celui des réseaux régionaux d'innovation, dans une perspective évolutive. L'exemple choisi concerne le développement récent des technologies permettant l'utilisation des doubles combustibles (éthanol/essence), dans la région de São Paulo (Brésil).

Mots-clés: Réseaux mondiaux, dynamiques régionales de connaissance, géographie économique évolutive, doubles combustibles, Brésil.

\section{INTRODUÇÃO}

Ao longo das duas últimas décadas, uma importante parte do trabalho em geografia económica e estudos regionais tem estudado as relações entre concentração espacial da actividade económica, inovação e desenvolvimento regional. Ao longo deste trajecto, conceitos como clusters (Porter, 1990), meios inovadores (Camagni, 1991; Maillat, 1995), regiões "aprendentes" (Asheim, 1996; Morgan, 1997) e, mais recentemente, sistemas regionais de inovação (Cooke, 1992, 2001; Asheim e Isaksen, 2002; Asheim e Gertler, 2005) têm tido bastante influência e encontram-se reflectidos nas agendas políticas e estratégias de desenvolvimento de muitas regiões.

Todos estes conceitos, de forma mais ou menos explícita, reconhecem a "região" (administrativa ou não) enquanto unidade territorial relevante, ao nível da qual se processam dinâmicas de interacção entre diferentes actores, facilitando decisivamente processos de criação de conhecimento, aprendizagem e inovação. A co-localização de empresas e outros actores (universidades, associações empresariais, centros tecnológicos e de I\&D, etc) é essencial para a geração espontânea de externalidades de conhecimento e de informação, conhecidas na literatura por buzz (Storper e Venables, 2004). Esta co-localização é também essencial para a coordenação das exigentes interacções inter organizacionais relacionadas com conhecimento e inovação, na medida em que facilita um entendimento conjunto, por via da presença de uma matriz institucional, social e cultural comum (Maskell e Malmberg, 1999). Em contexto de intensificação da globalização, este corpo de literatura tem decisivamente ajudado a perceber a resiliência da concentração espacial de actividades intensivas em conhecimento e inovação.

Ao mesmo tempo, diversos estudos no campo das ciências organizacionais e das cadeias de valor globais (Gereffi, et al., 2005; Berger, 2006) têm evidenciado esforços de inovação que ocorrem em redes de inovação de carácter transnacional. Conceitos como "inovação aberta" (Chesbrough, 2003) ou estratégias de inovação 
"meta-nacionais" (Doz et al., 2001) reflectem o reconhecimento de que muitos dos esforços de aquisição de conhecimento e inovação de empresas localizadas se processam cada vez mais por via de interacções deliberadas com outros actores localizados fora da "região" ou milieu inovador original. A crescente literatura em torno das "comunidades globais de conhecimento", quer epistemológica, quer de "prática", reflecte uma realidade similar (Amin e Cohendet, 2004).

Neste contexto, muito do trabalho recente da geografia económica tem sido feito no sentido de integrar na literatura este nexo global-local das dinâmicas de interacção na produção de conhecimento e inovação (Coe e Bunnell, 2003; Asheim e Coenen, 2005). Ao conciliar as vantagens da aprendizagem localizada com a relevância do acesso a redes globais de conhecimento e inovação, é influente a concetualização de Bathelt et al., (2004), que introduz a noção de "pipelines" - canais através dos quais actores geograficamente localizados acedem deliberadamente a conhecimento global - e buzz - referente a externalidades de conhecimento e informação resultantes da interacção co-localizada entre actores. Esta capacidade de combinar o "melhor de dois mundos" é apresentada na literatura como motor essencial de diferenciação competitiva das regiões em contexto de globalização: ... high levels of buzz and many pipelines may provide firms located in outward looking and lively clusters with a string of particular advantages not available to outsiders (Bathelt et al., 2004, p. 31).

Apesar de inspirador, uma das limitações que pode ser apontada a este corpo da literatura passa pela reduzida consideração dos efeitos dinâmicos resultantes da inserção de empresas (e a nível agregado, de clusters ou de regiões) em redes globais de conhecimento e inovação. Ao aceder a novas fontes de conhecimento, uma empresa pode ver alteradas, por exemplo, as suas rotinas instaladas de inovação empresarial e, de forma dinâmica, a natureza das suas competências tecnológicas e de conhecimento (Zollo e Winter, 2002). Em resultado, a alteração dinâmica da base de competências empresariais poderá ter efeitos na estrutura de diferentes redes inter-organizacionais localizadas, para além da geração de processos de co-evolução e adaptação institucional (Boschma, 2004). Este debate assume especial relevância quando as políticas públicas de inovação e competitividade a nível regional procuram cada vez mais complementar a criação de diversas plataformas, pólos e clusters com a inserção dos seus actores em redes globais (incentivos à internacionalização, estratégias de cooperação, atracção de investimento estrangeiro intensivo em conhecimento, etc.).

Este artigo, posicionando-se no quadro de uma abordagem evolucionista da geografia económica (Boschma e Frenken, 2006), pretende contribuir para este debate por duas vias. Por um lado, combinando desenvolvimentos recentes da geografia económica e estudos organizacionais, apresenta-se uma conceptualização dos efeitos dinâmicos resultantes da inserção em redes globais de conhecimento e inovação ao nível i) da empresa, nomeadamente pela especialização da base de conhecimento, ii) da rede de interacções regionais, por exemplo pela busca de parceiros para complementar a nova base de conhecimento e iii) da co-evolução institucional associada. Num segundo momento, o artigo ilustra esta 
conceptualização com o estudo do processo de desenvolvimento de um portfólio de novas tecnologias para o sector automóvel em São Paulo, associado aos sistemas bi-combustível, que permitem que um veículo automóvel se desloque a gasolina, etanol ou combinações dos dois. Trata-se de um processo marcado pela inserção dos seus principais actores e inventores em redes globais de inovação, com consequências ao nível da sua especialização empresarial e da emergência de novas dinâmicas de inovação regionais em torno de um portfolio de novas tecnologias "verdes" (Burtis et al., 2004).

O artigo está organizado da seguinte forma. A secção II revê o papel das interacções distantes nos processos de inovação localizada e os processos de evolução de clusters ao longo do tempo. A secção III apresenta um enquadramento dos efeitos dinâmicos associados à inserção de actores regionais em redes externas de inovação e conhecimento. A secção IV ilustra a conceptualização anterior com o processo de desenvolvimento de tecnologias bi-combustível em São Paulo, focando a análise nas redes globais de conhecimento envolvidas e na evolução regional associada. A secção $\mathrm{V}$ discute questões em aberto e implicações de política, nomeadamente regional.

\section{REVISITANDO A INOVAÇÃO LOCALIZADA: DINÂMICAS NO ESPAÇO E NO TEMPO}

Durante a última década, um importante corpo da geografia económica tem-se dedicado a estudar, por um lado, i) o papel de contactos e interacções externas à região no desenvolvimento de processos de inovação localizada e, por outro, ii) os processos de evolução dinâmica de clusters e especialização regional ao longo do tempo. Sem pretender ser exaustiva, esta secção revê alguns dos desenvolvimentos teóricos e empíricos mais relevantes, integrando-os de seguida (secção III) num modelo de análise das dinâmicas regionais determinadas pela integração dos seus actores em redes globais de conhecimento e inovação.

\section{Contactos externos e inovação localizada}

Uma das características centrais da literatura que aborda os processos de inovação localizada, em clusters de actividades relacionadas (Porter, 1990, 2000; Becatini, 1990; Maskell, 2001) é a alegada relevância dada à co-localização espacial de empresas e de outras entidades (universidades, agências e associações de suporte, etc.). De acordo com estas teorias, a proximidade geográfica ${ }^{2}$, ao

2 De forma mais ou menos explícita, estas teorias consideram que a co-localização geográfica coincide com (ou pelo menos implusiona) a presença de proximidade institucional, cultural e social entre os agentes. Para uma abordagem focada na separação destas diferentes dimensões de proximidade, ver Boschma (2005) ou Torre e Rallet (2005). 
reduzir significativamente custos de coordenação e transacção generalizados entre parceiros (Williamson, 1987), facilita a transmissão de conhecimento (tácito) e o desenvolvimento de esforços conjuntos de inovação atendendo à componente de sociabilidade associada a muitas das actividades envolvidas nos processos de inovação (Lundvall e Johnson, 1994).

Todavia, literatura recente em geografia económica, estudos regionais e organizacionais tem assinalado que muitos dos contactos desenvolvidos por empresas e outras organizações fora do seu "milieu" podem ser essenciais aos seus processos de aquisição de conhecimento, aprendizagem e inovação (Asheim e Isaksen, 2002; Amin e Cohendet, 2004; Asheim e Coenen, 2005; McKinnon et al., 2002; Vale e Caldeira, 2007; Wolfe e Gertler, 2004). Podem ser identificados pelo menos dois ramos de investigação, relacionados, que salientam esta realidade: em primeiro lugar, o trabalho em torno das cadeias de valor e redes de inovação global (Gerefi et al., 2005), salientando o papel de estratégias empresariais transnacionais no desenvolvimento de relacionamentos inter e intra empresariais, à escala global (Coe e Bunnell, 2003; Dicken et al., 2001); em segundo lugar, os estudos derivados das noções de comunidades de prática e epistémicas (Wenger, 1998; Knorr Cetina, 1999) recentemente trabalhados na geografia económica no estudo de formas temporárias de co-localização ao serviço da troca e produção de conhecimento (Maskell et al., 2006; Gertler, 2008). Este corpo de literatura identifica e explica o funcionamento de diversas formas interactivas de troca de conhecimento - por exemplo feiras, conferências, clusters temporários para o desenvolvimento de novos produtos, etc. - que, apesar de requererem contacto cara-a-cara e períodos de forte interacção, podem ser desenvolvidas com um carácter temporário, numa estrutura que se dissolve posteriormente. Na indústria automóvel, construção naval ou maquinaria especializada (Van Winden et al., 2008) é hoje em dia muito frequente a existência de plataformas temporárias para o desenvolvimento de um novo modelo ou de uma nova tecnologia, juntando competências de fornecedores independentes ou de diferentes departamentos de I\&D de um grupo transnacional (Gereffi, 2005; Birkinshaw e Hood, 1998). Este nexo local-global é uma realidade em muitos outros sectores e domínios de actividade, como nos mostram diversos casos, que vão desde a biotecnologia (Moodysson, 2008) aos media e indústrias criativas (Bathelt, 2005; Bathelt e Graf, 2008).

A recente conceptualização "local-global" em termos de buzz e pipelines (Bathelt et al., 2004) tenta reconciliar todo este novo corpo teórico e empírico revendo os anteriores conceitos de inovação localizada (Malmberg e Maskell, 2006), assinalando que o conhecimento, ainda que tácito, pode ser transmitido globalmente e que os processos de aprendizagem e inovação não são exclusivos de regiões inovadoras espacialmente delimitadas. Neste trabalho distingue-se entre os processos de aprendizagem que derivam da co-localização, por via de externalidades de conhecimento e informação - a que é chamado buzz (Storper e Venables, 2004) - e o conhecimento obtido e processado através de contactos externos e relações de actores locais com outros parceiros espalhados no globo, 
identificado como pipelines (Owen-Smith e Powell, 2004). A interacção virtuosa entre estas duas "fontes" de conhecimento, informação e colaboração tenderia a dotar clusters e regiões do "melhor de dois mundos", dando-lhes vantagens distintivas que se reforçariam cumulativamente ao longo do tempo (Giuliani, 2005; Bathelt, 2007).

\section{Evolução de clusters e regiões ao longo do tempo}

Existe hoje em dia um campo na geografia económica que estuda os processos de inovação regional numa perspectiva evolucionista ${ }^{3}$. No geral, esta perspectiva foca-se na relação interactiva entre estrutura e agência em diferentes contextos; o seu objectivo passa pela análise dos mecanismos sob os quais a acção humana (por exemplo empresas e suas estratégias), estruturas e instituições co-evoluem ao longo do tempo em diferentes contextos sócio-espaciais (Boschma e Lambooy, 1999).

Neste quadro de análise, um pressuposto importante é o de que o contexto territorial em que uma empresa opera exerce forte influência no seu comportamento - regiões e clusters são considerados como entidades relevantes, que afectam o comportamento das empresas e organizações que os constituem. Por um lado, empresas, regiões e clusters acumularam conhecimento e competências específicas e territorializadas (uma certa especialização, organização, competências e divisão de trabalho entre actores). Por outro, em linha com abordagens institucionalistas que estudam sistemas regionais de inovação (Cooke, 2001, Asheim e Gertler, 2005), estas entidades acumularam um conjunto de ambientes institucionais - normas, regulamentos, valores - e capital social (Saxenian, 1994; Morgan, 1997; Scott, 1998), que afectam decisivamente a intensidade e a natureza das relações na região, bem como a sua capacidade de aprendizagem, de re-invenção e desenvolvimento de novas actividades.

$\mathrm{Na}$ abordagem evolucionista a performance de um cluster ou região depende das suas capacidades dinâmicas de transferir conhecimento e gerar aprendizagem. Os mecanismos que suportam a coordenação dessas formas de interacção (mercados, redes inter-organizacionais) encontram-se mais ou menos enraizados num determinado ambiente institucional (Boschma, 2004). Estas atmosferas institucionais podem conduzir a excelentes dinâmicas de inovação (por exemplo, quando normas partilhadas e capital social entre os actores conduzem a atitudes de experimentação), mas podem também dificultar processos de desenvolvimento e inovação (por exemplo, quando baixos níveis de confiança ou bloqueios regulamentares impedem contactos fluidos e relações de mercado). Neste contexto, o desenvolvimento de um cluster ao longo do tempo é visto como o resultado da co-evolução dos seus activos específicos de competências e do seu ambiente

Para uma revisão sucinta da "evolução" recente ver, por exemplo, Boschma e Martin 
institucional. O desenvolvimento de um cluster é assim irreversível e dependente da sua história: após os acontecimentos (muitas vezes aleatórios) que determinam a sua nascença, cada cluster segue uma determinada trajectória de desenvolvimento (Boschma, 2004; Maskell e Malmberg, 2007).

"There exists a wide diversity of trajectories that differ with respect to which key organisations are involved (large firms, small firms, universities, public agencies), how knowledge is transmitted and diffused through the area (through inter-firm co-operation or through other means), which institutions affect the innovation process, and how institutions themselves are shaped, modified and transformed." (Boschma, 2004, p. 1008).

\section{REDES GLOBAIS E DINÂMICAS REGIONAIS: UMA LEITURA DINÂMICA}

Cruzando e integrando elementos das referências anteriores, nesta secção apresenta-se um modelo simplificado dos impactos dinâmicos resultantes da inserção dos actores de uma região ou cluster em redes globais ${ }^{4}$ de conhecimento e inovação. O modelo conceptualiza estes impactos ao nível i) da especialização das empresas envolvidas, ii) da evolução das suas redes regionais e iii) da co-evolução institucional associada (fig. 1).
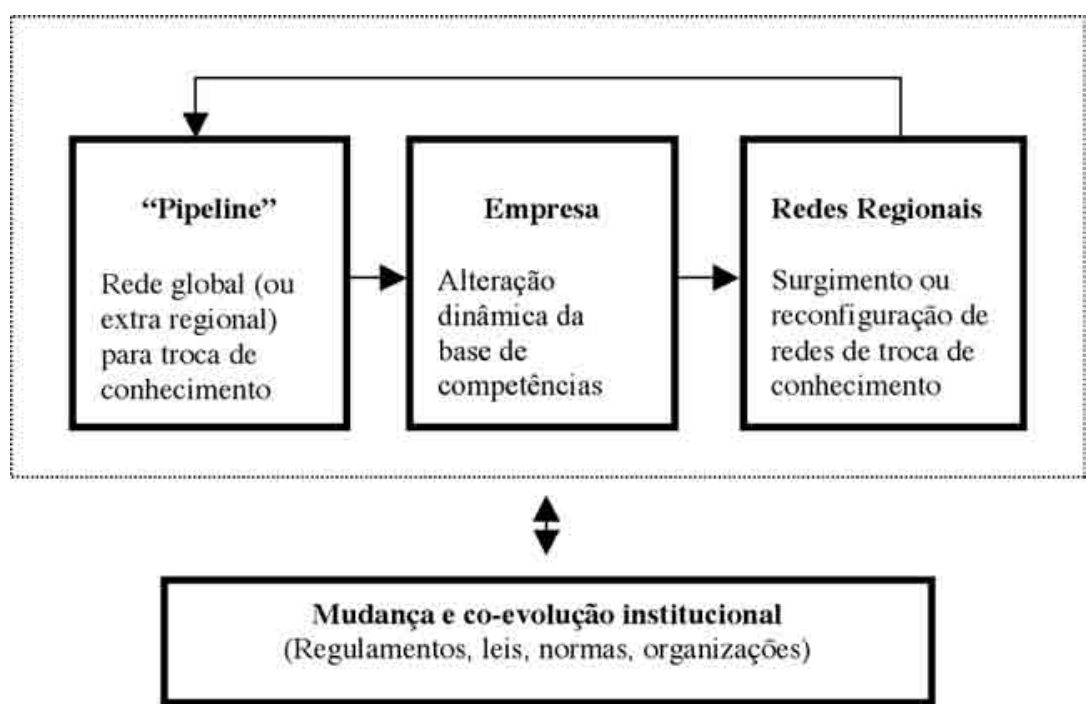

Fig. 1 - Redes globais e impactos regionais: uma conceptualização dinâmica.

Fig. 1 - Global networks and regional impacts: a dynamic framework.

4 Ou, mais concretamente, extra - "regionais". 
No modelo que se apresenta, redes globais de conhecimento e inovação (pipelines) são conceptualizadas como canais pelos quais se busca, desenvolve ou transfere conhecimento relacionado com tecnologias, produtos e negócios (Ahuja e Katila, 2001; Rosenkopf e Nerkar, 2001), susceptível de apropriação. Sendo uma actividade consumidora de recursos ${ }^{5}$ (Maskell et al., 2006), considerase que o estabelecimento de pipelines tem em vista uma certa estabilidade temporal, com possibilidades de repetição da interacção com os mesmos parceiros.

$\mathrm{O}$ argumento principal do modelo, detalhado nas secções seguintes, propõe que a inserção de uma empresa (ou, a nível agregado, de diversas empresas regionais) em pipelines, provoca efeitos dinâmicos na base de competências e de organização da empresa, isto é. mudança ou adaptação nas suas rotinas (Nelson e Winter, 1982). Este processo, por sua vez, acarreta efeitos de arrastamento, no sentido em que estas novas rotinas empresariais tendem a implicar também uma reconfiguração das respectivas redes inter-organizacionais de troca de conhecimento, inovação e buzz. Estes processos tenderão a co-evoluir com dinâmicas de adaptação institucional, gerando um feedback interactivo no sentido do reforço da (nova) base de competências e especialização regional (Maskell e Malmberg, 2007). As secções seguintes detalham sequencialmente os mecanismos propostos.

\section{Dinâmicas empresariais e de especialização}

O encetar de uma colaboração por via de uma pipeline, em última análise, representa a criação de proximidade "organizada" (Torre e Rallet, 2005) entre duas organizações ou actores separados no espaço. Tal pode suceder por diversas vias, em diferentes combinatórias de ecologias de projecto (Grabher, 2004), envolvendo ou não capital (ver secção 2.1 e também Rychen e Zimmermann, 2008, para uma descrição mais aprofundada de outros modos de pipelines).

Tal como referido, estes são mecanismos frequentemente utilizados por empresas globais com o objectivo de adquirir, desenvolver e explorar conhecimento disperso (Doz et al., 2001). No sentido de maximizar complementaridades, a inserção na rede tenderá a gerar especialização e divisão de trabalho entre os parceiros envolvidos, sendo este aliás o móbil principal de grande parte dos projectos inter-empresariais. Novos esforços de I\&D e de aquisição de conhecimento ao nível da empresa serão reorganizados, tendo em consideração as competências dos parceiros da rede, na direcção das combinações mais eficientes. A empresa ou estabelecimento local tenderá a especializar-se nas suas vantagens comparativas e competências chave, no sentido de tirar o máximo proveito do acesso aos recursos da rede global (Kogut e Zander, 2003).

Como resultado, uma empresa (ou um "nó" de uma rede global) tenderá a especializar-se em certas actividades, e a sua base de competências registará altera-

5 Por exemplo, identificação e selecção de parceiros, definição de condições de transferibilidade, desenvolvimento de confiança, etc. 
ções dinâmicas (Zollo e Winter, 2002): certas competências poderão ser canalizadas para outros pontos da rede (Cassiolato e Lastres, 2000), outras competências poderão surgir e outras ainda ser reforçadas ${ }^{6}$. De acordo com a literatura evolucionista (Nelson e Winter, 1982), está em causa um processo de mudança e redistribuição de rotinas empresariais, em resultado de um processo de selecção (de conhecimento, de parceiros) e de retenção (de novas rotinas numa unidade empresarial).

\section{Dinâmicas nas redes de inovação regionais}

Uma vez alterada a combinatória de competências e especialização tecnológica da empresa ou unidade empresarial são esperados ajustamentos dinâmicos nas suas redes e relacionamentos regionais, na procura de novos parceiros que complementem a nova base de competências, reconfigurando o seu eco-sistema local e regional de conhecimento e inovação ${ }^{7}$. Estas reconfigurações são consistentes com o trabalho recente de Hess (2004) e Jones (2008), que reconhecem a necessidade de uma abordagem que capture a dimensão dinâmica dos fenómenos de "embeddedness", resultantes da actividade dos agentes em diversas escalas espaciais. Nestes trabalhos, influenciados pelas abordagens sociológicas actor - rede (Law e Hassard, 1999), as redes de relacionamentos (por exemplo, para inovação) são vistas como uma realidade heterogénea, multidimensional e em mutação frequente ${ }^{8}$.

Assim, em primeiro lugar, são esperadas mudanças quantitativas e qualitativas na procura de qualificações por parte da empresa, com impactos nos mercados de trabalho local e regional. Por exemplo, as suas competências podem ser agora mais intensivas num dado tipo de produto ou serviço, requerendo competências e qualificações distintas das anteriores. Estas novas qualificações podem vir a ser encontradas na proximidade, ou numa região mais ampla (eventualmente não contígua), podendo alargar (ou reduzir) a configuração geográfica da bacia de emprego de referência. Em segundo lugar, de acordo com o novo perfil de competências da empresa, esta poderá necessitar de aceder a novas "atmosferas" de difusão de conhecimento e de interagir com novos parceiros, ao mesmo tempo que as competências de anteriores parceiros poderão perder relevância; simultaneamente, o conhecimento e informação com que a empresa contribui (competências, especialização, participação em nichos de mercado) são agora diferentes, com consequências nos tipos de buzz e difusão de conhecimento a nível regional. Finalmente, as novas competências centrais da empresa reque-

6 Por exemplo, no caso de uma empresa decidir especializar-se num certo grupo de competências, no sentido de tirar o maior proveito do acesso a uma rede de conhecimento externo, reforçando de forma dinâmica a sua capacidade de absorção (Cohen e Levintal, 1990).

7 Esta reorganização pode não suceder apenas a nível local ou regional pois a firma pode também procurar novos parceiros com competências complementares a nível global.

8 Para uma revisão detalhada desta literatura, suas aplicação na geografia económica e contraste com a versões mais convencionais inspiradas no trabalho de Granovetter (1985), ver Grabher (2006). 
rem novos parceiros complementares, podendo dar origem a novos consórcios e acordos de I\&D, outsourcing de serviços e produção, protocolos de cooperação com centros de I\&D regionais, etc. Van Winden et al. (2008) ilustra este fenómeno com a evolução dos acordos de investigação de vários centros de I\&D universitários em regiões de transição industrial, como o Norte de Portugal ou Ruhr area, na Alemanha - competências de investigação e acordos de I\&D, por exemplo nos domínios da metalurgia, perderam relevância para novos consórcios de I\&D no domínio dos novos materiais e energia, em função das novas necessidades de uma base empresarial regional em transformação.

As novas configurações e dinâmicas das redes regionais de conhecimento e inovação tenderão a determinar fenómenos de abertura de novas pipelines (fig.1), que podem suceder pelo menos por duas vias. Por um lado, por acção deliberada de parceiros externos, com o objectivo de aceder às novas dinâmicas localizadas de conhecimento e inovação, por via de uma dinâmica "de fora para dentro"; por outro, pela acção de actores locais procurando complementar a sua nova base de competências, no sentido de aceder a competências não disponíveis da região, ou simplesmente, de explorar conhecimento em outros pontos do globo (dinâmica de busca "de dentro para fora").

\section{Dinâmicas de co-evolução institucional}

De acordo com a leitura evolucionista, a alteração da combinatória de competências específicas regionais, em linha com Boschma (2004) e Maskell e Malmberg (2007) vem acompanhada e co-evolui com dinâmicas de mudança institucional ${ }^{9}$. A dinamização de pipelines e a emergência de um novo tipo de actividades e competências regionais impulsiona mudanças i) nos regulamentos e leis, por exemplo mudanças nos incentivos para a realização de I\&D pública e privada em determinados domínios; ii) nas normas, valores e hábitos, por exemplo no capital de confiança e interacção entre diferentes actores regionais e iii) no surgimento (ou adaptação) de organizações de suporte às novas competências regionais, como novas associações industriais e de desenvolvimento, parques tecnológicos e serviços de apoio, etc.

Num segundo momento, a presença destas novas instituições e organizações de suporte torna-se factor de atractividade para o reforço da (nova) base de competências regional, que evolui dinamizando a atracção de novas redes globais à região e o reforço de competências das suas empresas e redes. Segundo Maskell e Malmberg (2007):

9 É importante salientar que a geografia económica evolucionista não explica diferenças de performance e evolução regional por via de diferenças no quadro macro-institucional, mas por via de microhistórias e evoluções de firmas a operarem no contexto regional. Existe, assim, uma diferença chave face à abordagem institucionalista, em que o quadro institucional é por vezes considerado variável explicada e explicativa das diferenças e processos de desenvolvimento regional (Boschma e Frenken, 2006). 


\begin{abstract}
"National, regional or local institutions gradually develop over time in response to the special requirements of the presently dominating industry and lead to further specialization by creating a favourable environment for similar and complementary economic activity (...). Institutional adjustment gradually increases the fit with the chosen specialization and adds to the performance of the cluster" (p. 614).
\end{abstract}

\title{
IV. O CASO DAS TECNOLOGIAS BI-COMBUSTÍVEL EM SÃO PAULO
}

A presente secção ilustra o modelo apresentado desdobrando o processo de desenvolvimento das tecnologias bi-combustível em São Paulo, e consequente evolução regional em torno desta nova "especialização". Este estudo de caso foi desenvolvido durante o mês de Dezembro de 2007 e faz parte de um estudo comparativo internacional sobre dinâmicas de 10 clusters de base industrial em economias desenvolvidas e emergentes (para mais detalhes, ver Van Winden et al., 2008). Informação qualitativa primária foi recolhida no Estado de São Paulo, em diferentes cidades, por via de 25 entrevistas aprofundadas e semi-estruturadas, de duração compreendida entre 60-90 minutos, com directores de I\&D e gerentes de empresas e grupos transnacionais envolvidos na produção e desenvolvimento destas tecnologias, associações e federações industriais, centros de I\&D e representantes do poder político local e regional. Com vista a aumentar a fiabilidade, este leque de informação foi complementado com a análise de informação secundária, nomeadamente informação quantitativa e qualitativa proveniente de relatórios industriais, trabalhos de investigação prévia, relatórios de actividades recentes de empresas envolvidas, comunicações públicas, comunicados de imprensa, etc.

Antes de abordar a ilustração empírica importa fazer uma breve reflexão sobre o conceito de "região" utilizado no estudo. O Estado de São Paulo conta presentemente com cerca de 40 milhões de habitantes, metade dos quais na cidade e aglomeração metropolitana principal e o restante em torno de concentrações urbanas mais recentes, a menos de $100 \mathrm{~km}$ da cidade principal - caso de Campinas-Piracicaba, São José dos Campos e Sorocaba. Um diversificado leque de actividades industriais distribui-se hoje por estes pólos urbanos. Nas últimas duas décadas, o sector automóvel paulista desconcentrou-se significativamente da aglomeração principal em direcção a estes novos centros, que contam hoje não só com capacidade produtiva instalada, mas também com bases de conhecimento apreciáveis (centros tecnológicos e de I\&D universitários, mão-de-obra qualificada, etc.). Dado este pano de fundo, tornou-se difícil delimitar à priori qual a "região relevante" para a análise em causa, isto é, o processo dinâmico de desenvolvimento das tecnologias automóvel bi-combústivel.

Assim sendo, durante o trabalho de terreno associado a este estudo de caso optou-se por não estabelecer à priori uma delimitação rígida de "região", questionando os nossos interlocutores sobre os processos de desenvolvimento recente das suas actividades em "São Paulo". O objectivo deste procedimento era analisar qual a percepção dos entrevistados sobre a "região relevante" para as suas estratégias empresariais, projectos e interacções, nomeadamente para troca de 
conhecimento e informação. Da análise das entrevistas emergiu de forma clara a relevância da região constituída pela aglomeração principal e pelas novas aglomerações urbano-industriais mencionadas. Esta região (não contígua e radial) concentra o universo dos actores mais relevantes, possui uma elevada qualificação da mão-de-obra e é abrangida por um quadro macro-institucional bastante homogéneo, reconhecido como o mais avançado e estruturado do Brasil (por exemplo leis, regulamentos, incentivos para a Ciência e Tecnologia, organizações de suporte, etc.). Apesar de existirem algumas diferenças intra-região (nomeadamente no passado industrial e no activismo sindical), por simplificação e para efeitos desta ilustração, considera-se esta região não contígua como unidade relevante de análise - referida nas secções seguintes como "São Paulo", salvo indicação em contrário.

\section{Contexto do estudo de caso}

O Estado de São Paulo é o mais importante pólo económico do Brasil, representando em 2005 cerca de $40 \%$ do total do seu VAB - valor acrescentado bruto (IBGE, s.d.). A cidade de São Paulo, crescentemente terciarizada, concentra o maior número de sedes de empresas transnacionais na América Latina. Nos territórios envolventes encontra-se a base industrial mais diversificada do Brasil, incluindo sectores de forte conteúdo tecnológico, como a aeronáutica ou a biotecnologia; mais ainda, é responsável por cerca de $13 \%$ do VAB brasileiro no que toca a commodities e recursos naturais, como a cana-de-açúcar, laranja, soja ou café, para além de recursos energéticos como petróleo e gás natural.

A indústria automóvel tem quase um século de história em São Paulo (o Ford T foi o primeiro automóvel montado em São Paulo e no Brasil, em 1919), e é presentemente uma das suas indústrias mais dinâmicas. O sector desenvolveuse ao longo de diversos ciclos económicos e políticos, passando pela estratégia de substituição de importações, a partir dos anos 50, até à liberalização dos anos 90, com a redução progressiva de restrições às importações de automóveis e suas componentes. São Paulo concentra hoje estabelecimentos da grande maioria das construtoras automóvel multinacionais $(\mathrm{OEMs})^{10}$ e seus fornecedores, sendo destacadamente o maior pólo da indústria automóvel da América Latina.

Simultaneamente, o Estado de São Paulo concentra a maior parte da produção e transformação de cana de açucar do Brasil, cuja fermentação é famosa por dar origem ao álcool etanol, reconhecido como o bio-combústivel de maior qualidade e eficiência energética no globo (OECD, 2008). As primeiras experiências de automóveis movidos a álcool em São Paulo datam de 1920. Todavia, foi durante os anos 70, e em resposta ao elevado preço do petróleo e a diversos incentivos governamentais, que a produção de etanol disparou no Brasil. Em

10 OEM: Original Equipment Manufacturer. 
1986, mais de $90 \%$ dos automóveis brasileiros eram movidos a etanol combinado com gasolina (Teixeira, 2005).

Apesar da "crise do álcool" do início dos anos $90^{11}$, que determinou o retorno à hegemonia do uso da gasolina convencional, os crescentes "argumentos verdes" (Brilhante, 1997) e desenvolvimentos tecnológicos recentes trouxeram de volta o interesse no uso de etanol como alternativa à gasolina convencional. Avanços na biotecnologia da cana, juntamente com a mecanização do seu corte e a crescente eficiência energética da sua produção reanimaram o interesse público e privado no etanol e no seu potencial na substituição, pelo menos parcial, da gasolina.

É da parte dos fornecedores de sistemas automóveis que surge, no virar do século XX, uma das mais relevantes inovações incentivadoras do uso de etanol em automóveis. Em 2003 é lançado no Brasil o primeiro automóvel com tecnologia bi-combustível, isto é, com motor preparado para funcionar a etanol, gasolina ou qualquer combinação dos dois combustíveis, dando ao consumidor a liberdade de escolha, nomeadamente em função de flutuações do preço. Em 2007, todas as OEMs no Brasil produziam carros com esta tecnologia, totalizando 63 modelos diferentes (ANFAVEA, 2008). O quadro I ilustra o aumento exponencial da produção e adopção deste tipo de veículos no Brasil nos últimos anos, aproximando-se actualmente da totalidade dos automóveis produzidos, deduzidos das exportações.

Quadro I - Veículos automóveis produzidos no Brasil 1979-2007: motor a etanol, bi-combustível e total; milhares, anos seleccionados.

Table I - Car manufacturing in Brazil, 1979-2007: ethanol, flex-fuel and total; thousands, selected years.

\begin{tabular}{rrrr}
\hline & $\begin{array}{c}\text { Alcool E100 } \\
\text { (etanol) }\end{array}$ & Bi-combustivel & Total \\
\hline 1979 & 3 & 0 & 912 \\
1980 & 239 & 0 & 933 \\
1986 & 620 & 0 & 815 \\
1990 & 72 & 0 & 663 \\
1998 & 1 & 0 & 1254 \\
2000 & 9 & 0 & 1361 \\
2002 & 48 & 0 & 1521 \\
2003 & 32 & 40 & 1361 \\
2004 & 50 & 283 & 1863 \\
2005 & 43 & 776 & 2012 \\
2006 & 1 & 1249 & 2092 \\
2007 & 0 & 1717 & 2388 \\
\hline
\end{tabular}

Fonte: ANFAVEA (2008)

11 Resultante da queda do preço do petróleo e subida do preço do açúcar nos mercados internacionais, associado à redução drástica dos subsídios à produção de álcool (Shapiro, 1996). 
Nas secções seguintes, de acordo com o modelo detalhado na secção III, desdobra-se o processo evolutivo de desenvolvimento das tecnologias automóvel bi-combustível em São Paulo e o seu nexo global-local, nomeadamente no que toca ao acesso a plataformas globais de conhecimento (IV.2), especialização intra-empresarial (IV.3.1), dinâmicas nas redes de inovação regionais (IV.3.2) e co-evolução institucional (IV.3.3).

\section{O desenvolvimento das tecnologias flex-fuel no quadro de plataformas globais de conhecimento e inovação - pipelines}

Após a liberalização dos anos 90, OEMs e respectivos fornecedores investiram no aumento de capacidade produtiva e na modernização dos seus processos em diferentes aglomerações urbanas do Estado de São Paulo, quer entrantes transnacionais, quer empresas incumbentes (Mariotto, 2003). Tal como sugere a figura 2, este processo levou a alterações significativas da estrutura de capital no seio da indústria automóvel a operar em São Paulo, e no Brasil em geral (Shapiro, 1996). Tal sucedeu por via de diversas operações de take-over de empresas nacionais por grupos europeus e americanos, joint-ventures, investimentos de raiz de grupos transnacionais, bem como de falências de empresas incumbentes.

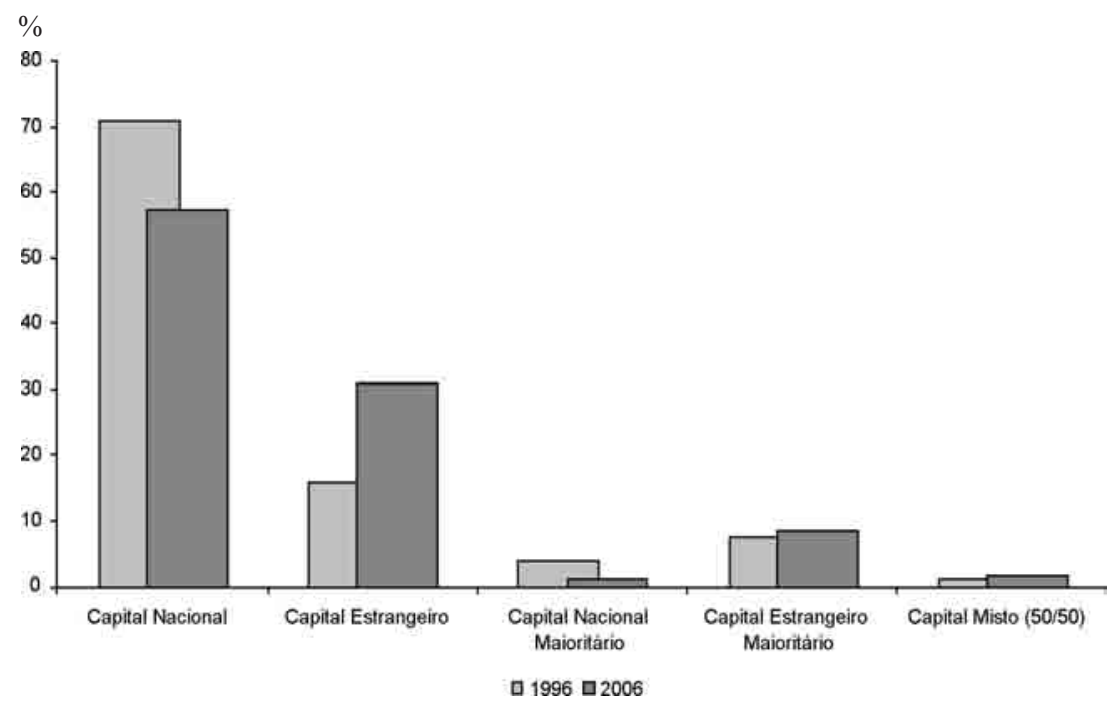

Fig. 2 - Fornecedores de componentes automóvel no Brasil, por origem do capital, \%. Fig. 2 - Automotive suppliers in Brazil, by origin of capital, $\%$.

Estes movimentos deram origem a uma estrutura de mercado caracterizada pela presença de estabelecimentos de grandes grupos transnacionais, a par de outros fornecedores de capital maioritariamente nacional, mais atomizados e, no 
geral, de menor capacidade técnico-financeira. No final dos anos 90 os processos de inovação localizada por via de interacções à la Porter e interacções de conhecimento são moderados e limitados à camada de empresas com maiores níveis de capacidade de absorção. A indústria automóvel em São Paulo, com o objectivo de servir o mercado brasileiro e latino-americano, explora cada vez mais tecnologia desenvolvida nas sedes e laboratórios de I\&D da tríade (Cassiolato e Lastres, 2000), por via de plataformas tecnológicas globais e de acesso restrito. As ligações a centros de conhecimento locais como universidades são praticamente nulas e os processos de co-desenvolvimento entre fornecedores transnacionais e empresas locais são residuais.

Neste contexto, o processo de desenvolvimento de novos sistemas de injecção de combustível (tecnologia central dos novos automóveis bi-combustível), entre meados dos anos 90 e inícios de 2000, surge num quadro de criação de plataformas globais de conhecimento e inovação - ou pipelines - de matriz intra-organizacional, entre engenheiros e departamentos de I\&D de grupos transnacionais espalhados no globo. Os grupos Robert Bosch (matriz alemã) e Magneti Marelli (matriz italiana) foram pioneiros no registo de patentes, seguidas da Delphi (matriz norte-americana), fornecendo actualmente toda a produção automóvel brasileira.

A Robert Bosch do Brasil, em Campinas, beneficiando de investigação prévia realizada nos laboratórios de I\&D nos Estados Unidos, registou a primeira patente global relacionada com as futuras tecnologias flex-fuel em 1988. Esta patente, co-desenvolvida por engenheiros brasileiros e americanos, registava a tecnologia de um sensor capaz de medir concentrações de etanol na gasolina por via do teor de oxigénio no tanque de combustível. A partir destes esforços, nos anos seguintes, engenheiros da unidade brasileira desenvolveram e testaram novos sensores para misturas de etanol e gasolina em equipas de projecto com engenheiros da matriz alemã - o objectivo passava por estabilizar parâmetros de referência, bem como reduzir o custo ainda avultado dos sensores (Teixeira, 2005). Em meados dos anos 90, a Magneti Marelli brasileira (Hortolândia - Campinas) enceta esforços no sentido de evitar o sistema de sensores físicos, baseando a sua tecnologia bi-combustível numa configuração de software directamente ligada ao sistema de injecção. As inovações associadas desenvolvem-se através de equipas de projecto com engenheiros italianos e brasileiros, apesar de grande parte do know-how sobre bio-combustíveis pertencer já à equipa brasileira.

Durante e após a adopção inicial dos novos sistemas bi-combustível, muitas outras empresas transnacionais em São Paulo estabeleceram pipelines globais de carácter semelhante (por exemplo diversos projectos de I\&D temporários intra-organizacionais), no sentido de desenvolver tecnologias complementares - carburadores, tanques anti-corrosão, bombas de combustível, reguladores de pressão do combustível, filtros, sistemas de arranque a frio, etc.

Assim, encontramos os grupos transnacionais de fornecedores de sistemas automóvel como iniciadores deste processo de desenvolvimento tecnológico, em articulação organizacional com outros estabelecimentos globais da corporação. 
Importa salientar que este processo é decisivamente facilitado pela presença de factores locais - por exemplo, a oferta de engenheiros e técnicos qualificados em São Paulo desde há várias décadas. Este factor é igualmente facilitador das dinâmicas regionais descritas nas secções seguintes.

\section{Dinâmicas regionais induzidas de conhecimento e inovação}

\subsection{Dinâmicas empresariais e de especialização}

$\mathrm{O}$ acesso a redes globais de inovação por parte de diferentes players do sector automóvel em São Paulo, intensificado a partir de meados dos anos 90, gerou alterações dinâmicas ao nível das suas rotinas e competências. Algumas das competências anteriores foram sendo esvaziadas para centros de I\&D da tríade (Cassiolato e Lastres, 2000), enquanto outras foram surgindo e outras reforçadas em função das vantagens relativas de cada unidade empresarial (incluindo potencial de acesso a conhecimento localizado).

O quadro II ilustra esta realidade, com exemplos de evolução de competências e esforços de I\&D de unidades brasileiras de diferentes tipos de fornecedores transnacionais de tecnologia automóvel ao longo da última década. Nestes exemplos existe um denominador comum: crescente especialização em diferentes pontos das cadeias intra-organizacionais de conhecimento global, associadas a divisão de trabalho e mudança de rotinas ao nível da unidade empresarial.

Quadro II - Investimentos em I\&D em estabelecimentos locais de empresas transnacionais (década de 1990 e 2007).

Table II $-R \& D$ investments by the local offices of transnational corporations (90's and 2007).

\begin{tabular}{lll}
\hline \multicolumn{1}{c}{ Empresa / estabelecimento local } & \multicolumn{1}{c}{ década de 1990 } & \multicolumn{1}{c}{ em 2007 } \\
\hline $\begin{array}{l}\text { "Empresa A" } \\
\begin{array}{l}\text { Fornecedor transnacional dos EUA, } \\
\text { líder em sistemas de distribuição } \\
\text { de combustível e componentes }\end{array}\end{array}$ & $\begin{array}{l}\text { Plásticos (geral), sistemas de } \\
\text { distribuição de combustível }\end{array}$ & $\begin{array}{l}\text { Novos materiais anti-corrosão } \\
\text { para bio-combustíveis; sistemas } \\
\text { de distribuição de misturas de } \\
\text { combustível. }\end{array}$ \\
$\begin{array}{l}\text { "Empresa B" } \\
\begin{array}{l}\text { Empresa brasileira de filtros automóvel, } \\
\text { adquirida por um grupo transnacional } \\
\text { italiano }\end{array}\end{array}$ & $\begin{array}{l}\text { Filtros e sistemas de filtragem } \\
\text { para ar, gasolina, etanol } \\
\text { e diesel }\end{array}$ & $\begin{array}{l}\text { Filtros e sistemas de filtragem } \\
\text { para etanol, bio diesel } \\
\text { e misturas }\end{array}$ \\
$\begin{array}{l}\text { "Empresa C" } \\
\text { Fornecedor alemão líder em sistemas } \\
\text { electrónicos e motores }\end{array}$ & $\begin{array}{l}\text { Sistemas para motores } \\
\text { a gasolina }\end{array}$ & $\begin{array}{l}\text { Sistemas para motores } \\
\text { bi-combustivel. }\end{array}$ \\
\hline
\end{tabular}

Fonte: elaboração própria, com base em trabalho de campo 
Actualmente, vários grupos transnacionais localizam em São Paulo pólos tecnológicos importantes no seio da sua estratégia empresarial global - estes pólos são crescentemente especializados, fazendo com que São Paulo se posicione como um centro de referência no uso e desenvolvimento de tecnologias ligadas aos bio-combustíveis. A Robert Bosch de Campinas conta hoje com cerca de 400 engenheiros a trabalhar em bio-combustíveis, no World Competence Centre for the Development of Conventional and Oxygen Fuel Systems. As suas competências há uma década eram mais diversificadas, mas bastante menos intensivas em tecnologia desenvolvida de raiz em São Paulo. A Magneti Marelli, também perto de Campinas, tem hoje em dia o maior centro de I\&D em combustíveis fora da Europa, estimando um crescimento de 30\% até 2010 .

Ao longo da última década, as mudanças no perfil de especialização e competências destas unidades locais têm já reflexo visível na evolução do padrão de exportações de componentes automóvel. Conforme ilustra o quadro III, a maioria das componentes e tecnologias associadas aos bio-combústiveis, tais como motores e sistemas associados, tiveram um crescimento muito mais significativo do que os outros tipos de produtos ao longo dos últimos anos.

Quadro III - Principais componentes automóveis exportadas pelo Brasil, 2000-2006. Table III - Main auto parts exported by Brazil, 2000-2006.

\begin{tabular}{|c|c|c|c|c|}
\hline Lugar & Componentes Exportadas & $\begin{array}{l}2000 \\
\text { milhões USD }\end{array}$ & $\begin{array}{l}2006 \\
\text { milhões USD }\end{array}$ & $\begin{array}{c}\text { Variação } \\
2000-06 \\
\%\end{array}$ \\
\hline 1 & $\begin{array}{l}\text { Outras partes e acessórios para veículos } \\
\text { automóveis }\end{array}$ & 443 & 1003 & 126 \\
\hline 2 & $\begin{array}{l}\text { Motores de ignição por centelha e combustão } \\
\text { interna }\end{array}$ & 106 & 599 & 452 \\
\hline 3 & Outros motores a diesel para veículos automóveis & 146 & 521 & 257 \\
\hline 4 & Blocos de cilindro, cabeçotes e cárteres & 123 & 462 & 276 \\
\hline 10 & $\begin{array}{l}\text { Injectores de combustível (incluindo bicos } \\
\text { injectores) }\end{array}$ & 55 & 198 & 261 \\
\hline 11 & $\begin{array}{l}\text { Injectores de combustível para motores de } \\
\text { compressão }\end{array}$ & 129 & 187 & 46 \\
\hline 13 & Pistões & 73 & 132 & 82 \\
\hline 16 & $\begin{array}{l}\text { Outros motores de ignição por compressão (diesel } \\
\text { ou semi-diesel) }\end{array}$ & 20 & 108 & 447 \\
\hline 19 & $\begin{array}{l}\text { Motores de pistão, de ignição por compressão } \\
\text { (diesel ou semi-diesel) }\end{array}$ & 12 & 100 & 822 \\
\hline
\end{tabular}

Fonte: Sindipeças, 2007 (elaboração do autor) 


\subsection{Dinâmicas nas redes de inovação regionais}

A especialização de muitos estabelecimentos na envolvente de São Paulo, em torno de tecnologias e soluções bi-combustível, está associada à emergência na região de novas parcerias regionais para troca de conhecimento e co-desenvolvimento de tecnologia. Após a abertura de pipelines, controlada por um número restrito de actores de dimensão quase exclusivamente transnacional, começam a ser visíveis interacções relevantes, típicas dos modelos de aprendizagem e inovação localizada (Maskell, 2001). Todavia, a embeddedness destas novas redes pode ser entendida como tendo um carácter fortemente determinado pela dinâmica de agentes individuais, actuando em escalas espaciais diferentes, e não propriamente vinculados a uma estrutura espacial de contactos e relações (Hess, 2004; Jones, 2008), que apenas recentemente começa a emergir.

Alguns exemplos ajudam a ilustrar o fenómeno. A TI Automotive do Brasil, especialista em sistemas de distribuição de combustível, com três unidades no Estado de São Paulo, trabalha conjuntamente com a Ford para ajustar as especificações dos seus sistemas e tanques de combustível aos requerimentos do etanol e, cada vez mais, de diferentes modalidades de bio-diesel. A Magneti Marelli passou a organizar em 2006 uma plataforma tecnológica com 13 fornecedores brasileiros (na maioria localizados em São Paulo) para a sua linha de montagem de bicos injectores de combustível, um dos seus produtos de mais elevada tecnologia aos quais é dada a possibilidade de fornecer outros estabelecimentos da Magneti Marelli nos Estados Unidos.

Noutras situações são os produtores de bio-combustíveis que dinamizam projectos locais de desenvolvimento tecnológico, testes de combustíveis e tecnologia automóvel. O projecto "Mistura 20", por exemplo, associa a Bertin (bio-combustíveis) com a Volkswagen bus, a Robert Bosch e a Cummins Engine Company (empresa americana especializada em motores a diesel) para testes conjuntos de qualidade de bio-combustíveis, parâmetros de mistura, bicos injectores e motores. Por outro lado, iniciativas de cooperação público-privado começam a surgir entre empresas, universidades e centros públicos de I\&D. A título de exemplo, i) a TI Automotive, o centro de I\&D do Ministério da Agricultura brasileiro e fornecedores locais cooperam para o desenvolvimento de bio-plásticos anti-corrosão; ii) a Universidade de São Paulo e a de Campinas associaramse numa parceria com a Delphi para novos desenvolvimentos tecnológicos ligados ao bi-combustível.

Estes exemplos espelham o surgimento de novos tipos de troca de informação e conhecimento a nível regional, isto é, novos tipos de buzz, que surgem praticamente de raiz em torno do ecossistema associado às tecnologias bi-combustível e bio-combustíveis, como se viu, tomando lugar em escalas espaciais variáveis e por vezes não contíguas. A estas novas dinâmicas está associado também um mecanismo de feedback de abertura e estabelecimento e novas pipelines. Um exemplo de "dentro para fora" é o caso da participação de fornecedores especializados da Magneti Marelli em diferentes plataformas nos Esta- 
dos Unidos, para exploração de tecnologias desenvolvidas em São Paulo; na óptica "de fora para dentro" são exemplos as diversas ecologias de projecto entre departamentos de I\&D globais temporariamente localizadas entre Brasil e os outros nós das redes transnacionais, com o objectivo de aceder a conhecimento brasileiro relativo a tecnologia automóvel bi-combustível (como por exemplo por parte da Saab - Suécia, ou da General Motors - Detroit).

\subsection{Dinâmicas de co-evolução institucional}

Os processos de abertura de pipelines, especialização e dinamização de novas redes e atmosferas de troca de conhecimento (localizadas e não localizadas) co-evoluiram com alterações e adaptação do quadro institucional, a diferentes níveis. Tendo sido num primeiro momento activadas por processos de agência (abertura de pipelines e criação de novo conhecimento em grupos transnacionais), o quadro de instituições e organizações de suporte co-evoluiu no sentido de suportar e facilitar processos de criação de redes locais e ancoramento de conhecimento no Estado de São Paulo, hoje uma referência em tecnologia automóvel relacionada com bio-combustíveis.

No final da década de 90 em São Paulo, no que toca ao quadro institucional de suporte ao sector automóvel, não existe um sistema de interacções facilitadoras de trocas de conhecimento e eficiência colectiva entre os actores do sector. As organizações de suporte são de carácter nacional, ainda que localizadas na cidade de São Paulo. Apesar da tradição industrial, o capital social é considerado bastante baixo e as interacções reduzidas. Os incentivos e regulamentos fiscais para tracção de investimento desfavorecem São Paulo em relação a outros Estados, e os apoios públicos para a ciência e tecnologia são organizados a nível Federal e não acessíveis ao sector privado. $\mathrm{O}$ factor mais distintivo face ao resto do Brasil é nesta altura a presença de uma certa cultura técnica e de uma classe de engenheiros brasileiros activa e organizada, centrada em São Paulo. A Ordem dos Engenheiros Brasileiros sedeada em São Paulo é geralmente reconhecida como uma plataforma informal de troca de conhecimento e desenvolvimentos técnicos, nomeadamente automóvel.

Não é todavia razoável considerar que o quadro macro-institucional de partida explicou a emergência e os desenvolvimentos tecnológicos em torno das tecnologias bi-combustível em São Paulo, apesar do contexto de liberalização da economia brasileira ter permitido e facilitado o processo de abertura a redes globais de inovação, bem como a oferta de engenheiros qualificados. Os primeiros fenómenos de co-evolução institucional surgiram em resposta aos esforços tecnológicos de grupos transnacionais localizados em São Paulo no desenvolvimento dos primeiros sistemas bi-combustível. Motivado pelo trabalho em curso nestas ecologias de I\&D privada, o Instituto de Pesquisa Tecnológica de São Paulo (IPT) do Governo Estadual organizou em 1998 um seminário de grande impacto na difusão do potencial das tecnologias bi-combustível na indústria automóvel, associando pela primeira vez as OEMs no desenvolvimento conjunto 
dos sistemas nos seus motores. Em 2000, a Associação Brasileira de Produtores de Cana de Açúcar, a petrolífera nacional Petrobrás e o IPT analisaram os impactos sociais, económicos e ambientais da tecnologia bi-combustível e, em 2002, o Governo Federal lançou o enquadramento de benefícios fiscais e incentivos à produção de veículos bi-combustível. Este movimento convenceu definitivamente as OEMs na adopção da tecnologia, dinamizando todo um leque de parcerias OEM-fornecedores de sistemas no desenvolvimento de variados modelos de veículos bi-combustivel.

Face ao sucesso da tecnologia e ao posicionamento de São Paulo como $h u b$ global (em função da crescente especialização intra-empresarial), o Estado de São Paulo lançou em 2006 um generoso pacote de incentivos à I\&D pública e privada em variados domínios relacionados com bio-combustíveis e tecnologias associadas, com destaque para os sistemas bi-combustível. Complementarmente, lançou um programa de intermediação tecnológica chamado "Tec-Days", enquanto plataforma facilitadora de futuros consórcios entre empresas e centros de I\&D. Por via de alguns destes incentivos, o número de parcerias entre players do sector automóvel, e entre estes e os produtores de bio-combustíveis tem aumentado, dinamizando novas redes e consórcios de inovação a nível regional. Não se traduzindo numa política de cluster "tradicional" (Tödtling e Trippl, 2005), estes incentivos têm contribuído para uma interpenetração de esforços tecnológicos em formato de plataforma, explorando complementaridades inter sectoriais e bases de conhecimento relacionadas, contribuindo para a criação de vantagem comparativa regional (Asheim, Boschma e Cooke, 2008).

O processo de co-evolução institucional, que solidificou e gerou nova interpenetração entre o sector automóvel e o dos bio-combustíveis, levou recentemente a investimentos do Estado de São Paulo na infra-estrutura física e organizacional do pólo de competitividade dos bio-combustíveis, perto da aglomeração urbana de Campinas. Esta nova infra-estrutura, em desenvolvimento, agirá como think-tank e facilitador de novas parcerias inter-regionais, para além de funcionar como suporte a actividades de intermediação e troca de conhecimento entre os actores envolvidos, estrutura de financiamento de I\&D conjunta e plataforma de suporte à definição de políticas públicas.

\section{DISCUSSÃO E CONCLUSÕES}

Ao longo das secções anteriores, o artigo desdobrou as fundações teóricas de uma conceptualização de inspiração evolucionista (Boschma e Martin, 2007) relativa aos processos resultantes da participação de actores locais em redes globais de conhecimento e inovação. O modelo apresentado foi ilustrado com as diferentes etapas inter-relacionadas na emergência das tecnologias bi-combustível em São Paulo, um processo marcado pela inserção dos seus principais actores em redes globais, intra-organizacionais, de conhecimento e inovação. 
As contribuições e argumentos apresentados podem ser sumariados em dois pontos principais. Em primeiro lugar, a conceptualização apresentada sugere que algumas micro tendências observadas ao nível da unidade empresarial, como por exemplo a participação em pipelines de conhecimento e inovação, ao fazerem alterar rotinas ao nível da empresa local, podem derivar em processos complexos de mudança a níveis mais agregados (por exemplo, nas redes de inovação regionais e quadro institucional), que se desviem das trajectórias prévias de especialização tecnológica regional - ou as façam emergir, como no caso das tecnologias bi-combustível em São Paulo. A apresentação destes mecanismos e a evidência de suporte contribui para aprofundar a conceptualização de local buzz-global pipelines de Bathelt et al. (2004), dando-lhe um carácter mais dinâmico.

Em segundo lugar, ao trabalhar no interface entre a geografia económica institucionalista e evolucionista (Boschma e Frenken, 2006), o artigo contribui para integrar e perceber os fenómenos de co-evolução institucional associados à emergência de um novo quadro tecnológico. $\mathrm{O}$ caso das tecnologias bi-combustível espelha o processo de emergência de um novo quadro institucional e organizacional a diferentes escalas, a partir do processo de abertura de pipelines, encetado pela acção de actores individuais (grupos transnacionais de sistemas a operar em São Paulo). Os estádios seguintes ilustram claramente um processo de interacção entre agência e estrutura institucional e organizacional (Boschma, 2004), no sentido da criação de um círculo virtuoso de especialização e excelência regional em torno da tecnologia automóvel associada aos bio-combustíveis, reforçando as competências e cultura técnica da engenharia paulista em torno destas especializações.

$\mathrm{O}$ argumento e a análise avançados neste artigo deixam algumas questões em aberto. Por um lado, a conceptualização apresentada carece ainda de mecanismos teóricos de moderação dos processos descritos, que permitam explicar melhor em que situações será mais provável a ocorrência dos fenómenos descritos, como por exemplo a especialização em torno de novas rotinas empresariais e a emergência de novas redes de inovação regionais. Por outro, o artigo sugere implicitamente dois fenómenos que carecem de conceptualização e validações empíricas mais aprofundadas. Em primeiro lugar, não existe ainda nem teoria nem testes empíricos suficientes associados aos fenómenos e impactos resultantes do cruzamento de dois sectores distintos numa "região", como por exemplo as tecnologias do sector automóvel e bio-combustíveis. Que impactos se podem esperar do cruzamento de percursos industriais distintos (Martin e Sunley, 2008)? Que tipos de sectores e de conhecimento devem estar presentes para a geração de percursos virtuosos? Por outras palavras: que tipos de "variedade" de bases de conhecimento se podem cruzar com vantagem (Frenken et al., 2007; Boschma e Iammarino, 2008)? Em segundo lugar, a dimensão espacial concreta na qual se processam os fenómenos de co-evolução merece uma atenção mais aprofundada. No caso de São Paulo, os processos observados derivaram em impactos espaciais intra-regionais - nomeadamente de redistribuição de activos de conhecimento e inovação inter aglomerações urbanas (por exemplo, 
das concentrações industriais tradicionais de São Paulo metropolitano para Campinas). Este processo de co-evolução entre dimensões espaciais, empresariais e organizacionais merece atenção mais aprofundada, e configura consequências políticas e de gestão urbana e intra-regional relevantes.

Não obstante a especificidade do caso em análise (quer pela sua dimensão quer pela presença de activos muito específicos, como os bio-combustíveis), a conceptualização apresentada incorpora implicações relevantes para a formatação de políticas públicas, nomeadamente a nível regional. Por um lado, alerta para a necessidade de prestar atenção a micro dinâmicas empresariais e aos efeitos que estas podem trazer ao nível de estruturas e redes regionais de inovação. Num contexto em que muitas políticas públicas a nível regional começam a complementar a implementação de clusters e sistemas de inovação (Doloreux e Parto, 2005) com o acesso de actores locais a redes globais de conhecimento (incentivos à internacionalização, estratégias de cooperação, plataformas internacionais de I\&D em redes de excelência, etc.), importa reflectir sobre os efeitos dinâmicos que se podem esperar da abertura dessas pipelines. Do mesmo modo que políticas orientadas para o cluster podem ter efeitos perversos e gerar lock-in (Todtling e Trippl, 2005), também políticas focadas na abertura de pipelines podem gerar efeitos indesejáveis, já que são susceptíveis de ter impactos ao nível da especialização de empresas locais e redes regionais de conhecimento e inovação. O caso de São Paulo ilustra impactos positivos da abertura de pipelines, embora seja possível imaginar casos em que tal fenómeno provoque o esvaziamento de competências regionais. Por exemplo, em situações em que a nova especialização empresarial e rotinas resultantes não encontrem parceiros complementares na região, levando à busca destas competências em outras regiões e a um afastamento progressivo entre as novas rotinas e a anterior base empresarial.

Por outro lado, os impactos dinâmicos da participação em pipelines podem derivar em novas necessidades de investimentos complementares a nível regional. Por exemplo, o fenómeno de emergência de uma nova especialização pode implicar necessidades de desenvolvimento de novas infra-estruturas físicas e organizacionais para acomodar e promover o seu desenvolvimento, como por exemplo parques tecnológicos, programas de formação específicos e outros incentivos financeiros, para I\&D em consórcio. Sempre que esta nova especialização se distancie de outros sectores da base económica regional, devem ser consideradas iniciativas de intermediação activa (Sverrisson, 2001; Mesquita, 2007) no sentido de uma evolução regional mais equilibrada e dinamicamente sustentável.

\section{AGRADECIMENTOS}

O autor agradece o apoio financeiro da FCT - Fundação para a Ciência e a Tecnologia (Bolsa de Doutoramento 36343 / 2007), a troca de opiniões frequentes sobre o tema deste artigo com Willem van Winden e o apoio do IHS - Institute for Housing and Urban Development Studies - no desenvolvimento do trabalho de campo associado a este estudo. 


\section{BIBLIOGRAFIA}

Ahuja G, Katila R (2001) Technological acquisitions and the innovation performance of acquiring firms: a longitudinal study. Strategic Management Journal, 22 (3):197-220.

Amin A, Cohendet P (2004) Architectures of knowledge: firms, capabilities, and communities. Oxford University Press, USA.

Anfavea (2008) Anuário da indústria automobilistica brasileira. São Paulo.

Asheim B, Boschma R, Cooke P. (2008) Constructing regional advantage: platform policies based on related variety and differentiated knowledge bases. Papers in Evolutionary Economic Geography, Utrecht University, Section of Economic Geography.

Asheim B, Coenen L, Moodysson J, Vang J (2007) Constructing knowledge-based regional advantage: implications for regional innovation policy. International Journal of Entrepreneurship and Innovation Management, 7 (2):140-155.

Asheim B, Gertler M (2005) The geography of innovation: regional innovation systems. In Fagerberg J, Mowery D, Nelson R (eds.) The Oxford Handbook of Innovation, Oxford University Press, Oxford: 291-317.

Asheim B T (1996) Industrial districts as 'learning regions': A condition for prosperity. European Planning Studies, 4 (4):379-400.

Asheim B T, Coenen L (2005) Knowledge bases and regional innovation systems: Comparing Nordic clusters. Research Policy, 34 (8):1173-1190.

Asheim B T, Isaksen A (2002) Regional innovation systems: the integration of local 'sticky'and global 'ubiquitous' knowledge. The Journal of Technology Transfer, 27 (1):77-86.

Bathelt H (2007) Buzz-and-pipeline dynamics: towards a knowledge-based multiplier model of clusters. Geography Compass, 1 (6):1282-1298.

Bathelt H (2005) Cluster relations in the media industry: exploring the 'distanced neighbour' paradox in Leipzig. Regional Studies, 39 (1):105-127.

Bathelt H, Gräf A (2008) Internal and external dynamics of the Munich film and TV industry cluster, and limitations to future growth. Environment and Planning A, 40:1944-1965.

Bathelt H, Malmberg A, Maskell P (2004) Clusters and knowledge: local buzz, global pipelines and the process of knowledge creation. Progress in Human Geography, 28 (1):31-56.

Becattini G (1990) The Marshallian industrial district as a socio-economic notion. In Pike F, Becattini G, Sengenberger W (eds.) Industrial districts and inter firm co-operation in Italy. International Institute for Labour Studies, Genova: 37-51.

Berger S (2006) How we compete: what companies around the world are doing to make it in today's global economy. Currency, Strawberry Hills.

Birkinshaw J, Hood N (1998) Multinational subsidiary evolution: capability and charter change in foreign-owned subsidiary companies. Academy of Management Review, 23 (4):773-795.

Boschma R (2005) Proximity and innovation: a critical assessment. Regional Studies, 39 (1):61-74.

Boschma R (2004) Competitiveness of regions from an evolutionary perspective. Regional Studies, 38 (9):1001-1014.

Boschma R, Iammarino S. (2008) Related variety, trade variety and regional growth in Italy. Papers in Evolutionary Economic Geography, Utrecht University, Section of Economic Geography.

Boschma R, Martin R (2007) Editorial: Constructing an evolutionary economic geography. Journal of Economic Geography, 7 (5):537. 
Boschma R A, Frenken K (2006) Why is economic geography not an evolutionary science? Towards an evolutionary economic geography. Journal of Economic Geography, 6 (3):273-302.

Boschma R A, Lambooy J G (1999) Evolutionary economics and economic geography. Journal of Evolutionary Economics, 9 (4):411-429.

Brilhante O (1997) Brazil's alcohol programme: from an attempt to reduce oil dependency in the seventies to the green arguments of the nineties. Journal of Environmental Planning and Management, 40 (4):435-449.

Burtis P, Epstein R, Hwang R (2004) Creating the California cleantech cluster. Natural Resources Defence Association, San Francisco, CA.

Camagni R (1991) Innovation networks: spatial perspectives. Belhaven Press, London and New York.

Cassiolato J E, Lastres H M M (2000) Local systems of innovation in MERCOSUR countries. Industry \& Innovation, 7 (1):33-53.

Chesbrough H W (2003) The era of open innovation. MIT Sloan Management Review, 44 (3):35-41.

Coe N M, Bunnell T G (2003) 'Spatializing'knowledge communities: towards a conceptualization of transnational innovation networks. Global Networks, 3 (4):437-456.

Cohen W M, Levinthal D A (1990) Absorptive capacity: a new perspective on learning and innovation. Administrative Science Quaterly, 35 (1):128-152.

Cooke P (2008) Regional innovation systems, clusters, and the knowledge economy. Industrial and Corporate Change, 10 (4):945-974.

Cooke P (1992) Regional innovation systems: competitive regulation in the New Europe. Geoforum, 23 (3):365-382.

Dicken P, Kelly P F, Olds K, Wai-Chung Yeung H (2001) Chains and networks, territories and scales: towards a relational framework for analysing the global economy. Global Networks: A Journal of Transnational Affairs, 1 (2):89-112.

Doloreux D, Parto S (2005) Regional innovation systems: Current discourse and unresolved issues. Technology in Society, 27 (2):133-153.

Doz Y L, Santos J, Williamson P (2001) From global to metanational: how companies win in the knowledge economy. Harvard Business School Press, Boston.

Frenken K, Van Oort F, Verburg T (2007) Related variety, unrelated variety and regional economic growth. Regional Studies, 41 (5):685-697.

Gereffi G, Humphrey J, Sturgeon T (2005) The governance of global value chains. Review of International Political Economy, 12 (1):78-104.

Gertler M (2008) Buzz without being there? Communities of practice in context. In Amin A, Roberts J (eds.) Community, economic creativity and organization. Oxford University Press, Oxford.

Giuliani E (2005) Cluster absorptive capacity: why do some clusters forge ahead and others lag behind? European Urban and Regional Studies, 12 (3):269.

Grabher G (2006) Trading routes, bypasses, and risky intersections: mapping the travels ofnetworks' between economic sociology and economic geography. Progress in Human Geography, 30 (2): 163 .

Grabher G (2004) Temporary architectures of learning: knowledge governance in project ecologies. Organization Studies, 25 (9):1491.

Grabher G (2002) The project ecology of advertising: tasks, talents and teams. Regional Studies: The Journal of the Regional Studies Association, 36 (3):245-262. 
Granovetter M (1985) Economic action and social structure: the problem of embeddedness. American Journal of Sociology, 91 (3):481.

Hess M (2004) 'Spatial'relationships? Towards a reconceptualization of embedded ness. Progress in Human Geography, 28 (2):165.

IBGE (2008) Regional acconts. São Paulo.

Jones A (2008) Beyond embeddedness: economic practices and the invisible dimensions of transnational business activity. Progress in Human Geography, 32 (1):71.

Knorr Cetina K (1999) Epistemic cultures: how the sciencesmake sense. Chicago University Press, Chicago.

Kogut B, Zander U (2003) Knowledge of the firm and the evolutionary theory of the multinational corporation. Journal of International Business Studies, 34 (6):516-529.

Law J, Hassard J (1999) Actor-network theory and after. Blackwell, Oxford.

Lundvall B, Johnson B (1994) The learning economy. Industry \& Innovation, 1 (2):23-42.

MacKinnon D, Cumbers A, Chapman K (2002) Learning, innovation and regional development: a critical appraisal of recent debates. Progress in Human Geography, 26 (3):293.

Maillat D (1995) Territorial dynamic, innovative milieus and regional policy. Entrepreneurship \& Regional Development, 7 (2):157-165.

Malmberg A, Maskell P (2006) Localized learning revisited. Growth and Change, 37 (1):1-18.

Mariotto F (2003) Flexibility and compromise in the Brazilian automotive industry. Third International Conference of the Iberoamerican Academy of Management São Paulo.

Martin R L, Sunley P J (2008) The place of path dependence in an evolutionary perspective on the economic landscape. In Boschma R, Martin R (eds.) Handbook of evolutionary economic geography. Edward Elgar, UK.

Maskell P (2001) Towards a knowledge-based theory of the geographical cluster. Industrial and Corporate Change, 10 (4):921-943.

Maskell P, Bathelt H, Malmberg A (2006) Building global knowledge pipelines: The role of temporary clusters. European Planning Studies, 14 (8):997-1013.

Maskell P, Malmberg A (1999) Localised learning and industrial competitiveness. Cambridge Journal of Economics, 23 (2):167-185.

Maskell P, Malmberg A (2007) Myopia, knowledge development and cluster evolution. Journal of Economic Geography, 7 (5):603.

Mesquita L F (2007) Starting over when the bickering never ends: rebuilding aggregate trust among clustered firms through trust facilitators. Academy of Management Review, 32 (1):72-91.

Moodysson J, Coenen L, Asheim B (2008) Explaining spatial patterns of innovation: analytical and synthetic modes of knowledge creation in the Medicon Valley life-science cluster. Environment and Planning A, 40 (5):1040.

Morgan K (1997) The learning region: institutions, innovation and regional renewal. Regional Studies, 31 (5):491-503.

Nelson R R, Winter S G (1982) An evolutionary theory of economic change. The Belknap Press, Cambridge, MA and London.

OECD (2008) Economic assessment of bio-fuel support policies. OECD, Paris.

Owen-Smith J, Powell W W (2004) Knowledge networks as channels and conduits: the effects of spillovers in the boston biotechnology community. Organization Science, 15 (1):5. 
Porter M E (2000) Location, competition, and economic development: local clusters in a global economy. Economic Development Quarterly, 14 (1):15.

Porter M E (1990) The competitive advantage of nations. Free Press, New York.

Rodriguez-Pose A, Arbix G (2001) Strategies of waste: bidding wars in the Brazilian automobile sector. International Journal of Urban and Regional Research, 25 (1):134-154.

Rosenkopf L, Nerkar A (2001) Beyond local search: boundary-spanning, exploration, and impact in the optical disk industry. Strategic Management Journal, 22 (4):287-306.

Rychen F, Zimmermann J B (2008) Clusters in the global knowledge-based economy: knowledge gatekeepers and temporary proximity. Regional Studies, 42 (6):767-776.

Saxenian A L (1994) Regional advantage: culture and competition in silicon valley and route 128. Harvard University Press, Harvard.

Scott A J (1998) Regions and the world economy: the coming shape of global production, competition, and political order. Oxford University Press, Oxford.

Shapiro H (1996) The mechanics of Brazil's auto industry. NACLA report on the Americas, Jan-Feb 2006.

Sindipeças (2007) Brazilian autoparts industry performance 2007. Sindipeças, São Paulo.

Storper M, Venables A J (2004) Buzz: face-to-face contact and the urban economy. Journal of Economic Geography, 4 (4):351-370.

Sverrisson Á (2001) Translation networks, knowledge brokers and novelty construction: pragmatic environmentalism in Sweden. Acta Sociologica, 44 (4):313-327.

Teixeira E T (2005) Flex fuel tecnology in Brasil. DNA Institute, São Paulo.

Tödtling F, Trippl M (2005) One size fits all? Towards a differentiated regional innovation policy approach. Research Policy, 34 (8):1203-1219.

Torre A, Rallet A (2005) Proximity and localization. Regional Studies, 39 (1):47-59.

Vale M, Caldeira J (2007) Proximity and knowledge governance in localized production systems: the footwear industry in the north region of Portugal. European Planning Studies, 15 (4):531-548.

Van Winden W, Van den Berg L, Carvalho L, Van Tuijl E (2008) The role of manufacturing in the new knowledge based urban economy. EURICUR, Erasmus University Rotterdam.

Wenger E (1998) Communities of practice: learning, meaning, and identity. Cambridge University Press, Cambridge.

Williamson O E (1987) The economic institutions of capitalism: firms, markets, relational contracting. Collier Macmillan Publishers, London.

Wolfe D A, Gertler M S (2004) Clusters from the inside and out: local dynamics and global linkages. Urban Studies, 41 (5):1071-1093.

Zollo M, Winter S (2002) Deliberate learning and the evolution of dynamic capabilities. Organization Science, 13 (3): 339-351. 